\title{
COMPACTLY GENERATED ALGEBRAS OVER DISCRETE FIELDS ${ }^{1}$
}

\author{
BY SETH WARNER
}

\author{
Communicated by R. Arens, October 10, 1966
}

The structure of locally compact vector spaces over complete division rings topologized by a proper absolute value (or, more generally, over complete division rings of type $V$ ) is summarized in the now classical theorem that such spaces are necessarily finite-dimensional and possess the cartesian product topology ([2], [5, Theorems 5 and 7], [1, pp. 27-31]).

Here we present some results on locally compact vector spaces and algebras over infinite discrete fields. Since any topological vector space over a topological division ring $K$ remains a topological vector space if $K$ is retopologized with the discrete topology, some restriction needs to be imposed, and the most natural restriction is straightness: A topological vector space $E$ over a topological division ring $K$ is straight if for every nonzero $a \in E, \lambda \rightarrow \lambda a$ is a homeomorphism from $K$ on to the one-dimensional subspace generated by $a$. Thus if $K$ is discrete, a straight $K$-vector space is one all of whose one-dimensional subspaces are discrete.

A category argument establishes the following theorem:

THEOREM 1. If $K$ is an infinite discrete field, a straight locally compact $K$-vector space of countable dimension is discrete.

A field $K$ is algebraic if it has prime characteristic and if it is an algebraic extension of its prime subfield.

THEOREM 2. If $K$ is an infinite discrete division ring, then there exists an indiscrete straight locally compact $K$-vector space if and only if $K$ is an algebraic field.

The proof depends on a theorem of Gleason [3, Lemma 1.4.2] concerning the existence of one-parameter subgroups in locally compact groups having no small subgroups and on Jacobson's theorem [4, p. 183] that algebraic division algebras over finite fields are commutative.

A theorem of Pontrjagin [7, p. 153] and Theorem 2 then establish that a straight locally compact vector space over an infinite discrete field is totally disconnected.

\footnotetext{
${ }^{1}$ Research supported by National Science Foundation grant GP-4473.
} 
The variety of locally compact spaces is indicated by the fact that if $K$ is a discrete infinite algebraic field, there is an indiscrete straight locally compact $K$-vector space $E$ each of whose finite-dimensional subspaces is discrete and whose topological dual $E^{*}$ is total, and there is also an indiscrete straight locally compact $K$-vector space $F$ that contains a dense two-dimensional subspace and admits no nonzero continuous linear forms.

A topological vector space is compactly generated if it contains a compact set of generators. The proof of the following theorem is immediate:

THEOREM 3. A straight locally compact vector space over an infinite discrete field is the topological direct sum of a compactly generated subspace and a discrete subspace.

Thus the topology of a straight locally compact space is of significance only on compactly generated subspaces. The extent of familiar algebras carrying straight locally compact topologies is suggested by the following theorem:

THEOREM 4. If $E$ is a straight locally compact vector space over an infinite discrete field $K$, then the $K$-algebra of all continuous linear operators on E, equipped with the compact-open topology, is a straight locally compact $K$-algebra if and only if $E$ is compactly generated.

The analogue of Theorem 3 fails for algebras, as Theorem 4 and the following theorem show:

Theorem 5. Let $K$ be an infinite topological field, and let $A$ be a locally compact primitive $K$-algebra that contains minimal left ideals. If $A$ is compactly generated, then $A$ is isomorphic to the $K$-algebra of all linear operators on a finite-dimensional vector space over a locally compact division ring $D$ that algebraically is an extension of $K$.

The proof is based on that part of Jacobson's theorem concerning primitive rings with minimal left ideals [4, pp. 75-77] which asserts that if $e$ is a primitive idempotent, multiplication is a nondegenerate bilinear form on $e A \times A e$, where $e A$ is regarded as a left and $A e$ a right vector space over $e A e$.

It is possible to give a fairly concrete description of commutative, semisimple, straight locally compact algebras over infinite discrete fields. Henceforth, $\Omega$ is the algebraic closure of the field $\boldsymbol{Z}_{p}$ of integers modulo $p$, and $K$ is an infinite subfield of $\Omega$, equipped with the discrete topology. We shall say that $\left(K_{\gamma}\right)_{\gamma \in \Gamma},\left(\Gamma_{n}\right)_{n \geqq 0},\left(F_{n}\right)_{n \geqq 0},\left(\sigma_{\gamma}\right)_{\gamma \in \mathrm{r}}$ is a family of $K$-ingredients if the following conditions hold: 
$1^{\circ}$. Each $K_{\gamma}$ is an extension field of $K$, and every element of $K_{\gamma}$ algebraic over $\boldsymbol{Z}_{p}$ belongs to $\Omega$.

$2^{\circ}$. $\left(\Gamma_{n}\right)_{n \text { z } 0}$ is a partition of the index set $\Gamma$.

$3^{\circ}$. For each $n \geqq 0$ and each $\gamma \in \Gamma_{n}, F_{n}$ is a finite subfield of $K_{\gamma}$, and $\sigma_{\gamma}$ is an automorphism of $F_{n}$.

$4^{\circ}$. For each $\alpha \in K$ there exists $m \geqq 0$ such that for all $n>m, \alpha \in F_{n}$ and $\sigma_{\gamma}(\alpha)=\alpha$ for all $\gamma \in \Gamma_{n}$.

Given a family of $K$-ingredients, we let $V=\left\{\left(x_{\gamma}\right) \in \prod_{\gamma \in \Gamma} K_{\gamma}\right.$ : there exists $\left(a_{n}\right) \in \prod_{n \geqq 0} F_{n}$ such that for every $n \geqq 0, \sigma_{\gamma}\left(a_{n}\right)=x_{\gamma}$ for all $\left.\gamma \in \Gamma_{n}\right\}$, and we let $A=\left\{\left(x_{\gamma}\right) \in \prod_{\gamma \in \Gamma} K_{\gamma}\right.$ : there exists $\left(a_{n}\right) \in \prod_{n \geqq 0} F_{n}$ such that for all but finitely many $n \geqq 0, \sigma_{\gamma}\left(a_{n}\right)=x_{\gamma}$ for all $\left.\gamma \in \Gamma_{n}\right\}$. Then $A$ is a commutative semisimple $K$-algebra, and $V$ is a subring of $A$ that generates $A$ as an ideal; equipped with the topology for which $\left\{\lambda_{1} V \cap \lambda_{2} V \cap \cdots \cap \lambda_{n} V: \lambda_{1}, \lambda_{2}, \cdots, \lambda_{n} \in K^{*}\right\}$ is a fundamental system of neighborhoods of zero, $A$ is an indiscrete straight locally compact $K$-algebra, and $V$ is a compact open subring of $A$. We shall call a subalgebra $B$ of $A$ a topological algebra defined by the family of $K$-ingredients $\left(K_{\gamma}\right)_{\gamma \in \Gamma},\left(\Gamma_{n}\right)_{n \geqq 0},\left(F_{n}\right)_{n \geqq 0},\left(\sigma_{\gamma}\right)_{\gamma \in \Gamma}$ if $B$ contains $V$ and if for every finite subset $\Delta$ of $\Gamma$ and every $\left(x_{\gamma}\right) \in A$, there exists $\left(y_{\gamma}\right) \in B$ such that $y_{\gamma}=x_{\gamma}$ for all $\gamma \in \Delta$; the algebra $A$ is called the full topological algebra defined by this family of $K$-ingredients.

THEOREM 6. If $A$ is an indiscrete straight locally compact $K$-algebra, then $A$ is commutative and semisimple if and only if $A$ is the topological direct product of a discrete commutative semisimple $K$-algebra and $a K$ algebra isomorphic to a topological algebra defined by a family of $K$ ingredients.

The proof is based on a theorem of Kaplansky [6, Theorem 12] and the theorem of Pontryagin, van Dantzig, and Jacobson that the topology of a locally compact field is defined by an absolute value. The latter theorem is used to show that if $A$ contains a compact open subring $V$ that generates $A$ as an ideal and if $\mathrm{m}$ is a closed regular maximal ideal of $A$, then $V \cap \mathfrak{m}$ is a regular maximal ideal of $V$. The corresponding statement for noncommutative algebras (where "regular maximal" is replaced either by "primitive" or by "regular maximal left") is not in general true, even if $\mathfrak{m}$ is open.

For compactly generated algebras we may make the description of Theorem 6 more precise:

THEOREM 7. If $A$ is an indiscrete straight locally compact $K$-algebra, then $A$ is commutative, semisimple, and compactly generated if and only if $A$ is isomorphic to the full topological algebra defined by a family of 
$K$-ingredients $\left(K_{\gamma}\right)_{\gamma \in \Gamma},\left(\Gamma_{n}\right)_{n \geqq 0},\left(F_{n}\right)_{n \geqq 0},\left(\sigma_{\gamma}\right)_{\gamma \in \Gamma}$ satisfying the following conditions:

$1^{\circ}$. For each $n \geqq 0, \gamma \rightarrow \sigma_{\gamma}$ is an injection from $\Gamma_{n}$ into Aut $F_{n}$; hence $\Gamma_{n}$ is finite for each $n \geqq 0$.

$2^{\circ}$. $K_{\gamma}=K\left(F_{n}\right)$ for all $\gamma \in \Gamma_{n}$, all $n \geqq 0$.

$3^{\circ}$. For each $n \geqq 0$, if $\alpha$ and $\beta$ are distinct members of $\Gamma_{n}$, then $K$ and $F_{n}$ are not linearly disjoint over the intersection of $K$ with the fixed field of $\sigma_{\alpha}^{-1} \sigma_{\beta}$.

\section{REFERENCES}

1. N. Bourbaki, Espaces vectoriels topologiques, Ch. I-II, Act. Sci. Ind. 1189, Hermann, Paris, 1953.

2. J. Braconnier, Sur les espaces vectoriels localements compacts, Comptes Rendus de l'Académie des Sciences, Paris 222 (1946), 777-778.

3. Andrew M. Gleason, Groups without small subgroups, Ann. of Math. 56 (1952), 193-212.

4. Nathan Jacobson, Structure of rings, Amer. Math. Soc. Colloq. Publ. Vol. 37, Amer. Math Soc., Providence, R. I., 1956.

5. Irving Kaplansky, Topological methods in valuation theory, Duke Math. J. 14 (1947), 527-541.

6. - Locally compact rings, Amer. J. Math. 70 (1948), 447-459.

7. L. Pontrjagin, Topological groups, Princeton Univ. Press, Princeton, N. J., 1946.

\section{DUKE UNIVERSITY}

